\title{
Fertility preservation before an ABVD protocol: no new evidence to support changing the recommendations
}

\author{
Catherine Poirot ${ }^{*, 1,2}$, Nathalie Dhedin' ${ }^{1}$ Pauline Brice ${ }^{3}$
}

First draft submitted: 30 September 2016; Accepted for publication: 23 November 2016; Published online: 6 December 2016

We have read with interest the article by Sonigo et al., entitled "History of ABVD alters the number of oocytes vitrified after in vitro maturation in fertility preservation candidates" [1].

This study compared the competence of oocyte to mature in vitro to mature oocytes collected for fertility preservation in two groups of patients. The group of interest comprised 22 patients who had previously undergone ABVD (doxorubicin, bleomycin, vinblastine and dacarbazine)-type chemotherapy for Hodgkin disease, including 13 patients presenting relapsed lymphoma and nine patients presenting breast cancer at the time of fertility preservation. The control group comprised patients presenting breast cancer and referred for fertility preservation; these patients had no previous history of chemotherapy (44 patients). Sonigo et al. reported that the rate of oocyte maturation in these two groups was identical.

However, although the patients of these two groups presented no significant differences in terms of age, BMI, anti-Müllerian hormone (AMH) level and antral follicle count, the authors highlight the fact that in both groups, the number of oocytes collected for fertility preservation was significantly lower in the population having already undergone ABVD chemotherapy, compared with the patients who had breast cancer, and question the recommendations, proposing instead fertility preservation prior to ABVD treatment.

We would like to discuss this result and these conclusions.

The result is surprising, since the literature presents several examples, even by the authors themselves, that there is a good correlation between the antral follicle count, AMH level and the number of oocytes collected [2] and that the two groups of patients in this study present no significant differences in terms of these parameters. It would, therefore, seem important to specify whether the oocyte retrievals were carried out by the same practitioner or by different practitioners. Different operators responsible for the oocyte retrieval could easily cause variations in results, because of the small study population. Furthermore, the authors' hypothesis to explain the smaller number of oocytes collected from women who had had ABVD, was the possibility of ovary fibrosis, described in the study by Meirow et al. [3], thus making sample collection more difficult. However, Meirow et al. did not make this claim in their study, since they were unable to link the lesions observed to a specific type of chemotherapy protocol and they did not find them in all the patients previously exposed to chemotherapy.

Furthermore, an important point not discussed in this article, is the impact of Hodgkin lymphoma on the ovarian function. It has been proved that Hodgkin

\section{KEYWORDS}

- ABVD • fertility preservation

- Hodgkin lymphoma 
lymphoma, at the time of the illness, has a negative effect on the parameters enabling evaluation of the ovarian reserve, due to a mechanism that has not yet been explained [4,5]. This effect results in a reduced response to ovulation stimulation and a smaller number of oocytes collected than for patients with breast cancer [4] or another cancer pathology [5].

Although all the patients had previously had ABVD chemotherapy, the group in this study is heterogeneous, since composed of patients presenting Hodgkin lymphoma relapse (13 patients) and patients with breast cancer (nine patients), at the time of fertility preservation. Can these patients be included in the same group, bearing in mind that the lymphoma itself affects the ovarian function?

In terms of the effect of ABVD on the ovarian function, monitoring studies of patients having undergone an ABVD protocol have shown the low impact of this chemotherapy on the ovarian reserve and fertility. In their study of AMH level variations, Decanter et al. reported that the AMH levels returned to their initial levels 1 year after the end of ABVD-type chemotherapy [6]. Clinically, as summarized in the review by Harel et al., in patients wanting to have a child, fertility rates are identical to those of the control groups [7]. In 2008, Rosendahl et al. studied the ovarian and procreative functions in patients having undergone a unilateral oophorectomy for fertility preservation. In the group of patients having received ABVD treatment only after the oophorectomy, the ovarian function was not affected in almost all patients, even though they only had one remaining ovary [8]. In our experience, in 123 patients aged between 18 and 35 years, with a diagnostic whose median monitoring duration is 7 years, $57 \%$ have had at least one pregnancy, even after the age of 40 years for some. Regarding the other patients, either they were not in a relationship or infertility was not mentioned in the file [Brice Pet al., Pers. Comm.].

Therefore, to distinguish between the effects of the ABVD protocol and the illness on the results of the Sonigo et al. study, it would have been interesting to know if, within the population of ABVD-treated patients, the 13 presenting relapsed Hodgkin disease at the time of fertility preservation had a smaller number of oocytes collected and a lower AMH dosage than the nine others.

Due to the impact of Hodgkin disease on the ovarian function, the low ovarian toxicity for the ABVD protocol attested by data from the literature, the heterogeneity of the group of patients studied, the small patient population of each group and the retrospective nature of the study, this study therefore provides no further solid evidence to support the ovarian toxicity of ABVD and it would appear to be quite precipitous to revise the recommendations in terms of fertility preservation for patients who are to be treated with this protocol.

\section{Financial \& competing interests disclosure}

The authors have no relevant affiliations or financial involvement with any organization or entity with a financial interest in or financial conflict with the subject matter or materials discussed in the manuscript. This includes employment, consultancies, honoraria, stock ownership or options, expert testimony, grants or patents received or pending, or royalties.

No writing assistance was utilized in the production of this manuscript.

\section{References}

1 Sonigo C, Seroka A, Cedrin-Durnerin I, Sermondade N, Sifer C, Grynberg M. History of $A B V D$ alters the number of oocytes vitrified after in vitro maturation in fertility preservation candidates. Future Oncol. 12(14), 1713-1719 (2016).

2 Sonigo C, Simon C, Boubaya M et al. What threshold values of antral follicle count and serum AMH levels should be considered for oocyte cryopreservation after in vitro maturation? Hum. Reprod. 31(7), 1493-1500 (2016).

3 Meirow D, Dor J, Kaufman B et al. Cortical fibrosis and blood-vessels damage in human ovaries exposed to chemotherapy. Potential mechanisms of ovarian injury. Hum. Reprod. 22(6), 1626-1633 (2007).

4 Lawrenz B, Fehm T, von Wolff M et al. Reduced pretreatment ovarian reserve in premenopausal female patients with Hodgkin lymphoma or non-Hodgkin-lymphoma evaluation by using antimüllerian hormone and retrieved oocytes. Fertil. Steril. 98(1), 141-144 (2012).

5 Lekovich J, Lobel ALS, Stewart JD, Pereira N, Kligman I, Rosenwaks Z. Female patients with lymphoma demonstrate diminished ovarian reserve even before initiation of chemotherapy when compared with healthy controls and patients with other malignancies. J. Assist. Reprod. Gent. 33, 657-662 (2016).
6 Decanter C, Morschhauser F, Pigny P, Lefebvre C, Gallo C, Dewailly D. AntiMüllerian hormone follow-up in young women treated by chemotherapy for lymphoma: preliminary results. Reprod. Biomed. Online 20(2), 280-285 (2010).

7 Harel S, Fermé C, Poirot C. Management of fertility in patients treated for Hodgkin's lymphoma. Haematologica 96(11), 1692-1699 (2011).

8 Rosendahl M, Andersen CY, Ernst E et al. Ovarian function after removal of an entire ovary for cryopreservation of pieces of cortex prior to gonadotoxic treatment: a follow-up study. Hum. Reprod. 23(11), 2475-2483 (2008). 